\title{
Polysèmes
}

Revue d'études intertextuelles et intermédiales

\section{Logique de l'après-coup : Chatterton et le contemporain}

The Logics of Afterwardsness: Presenting the Contemporary in Chatterton

Jean-Michel Ganteau

\section{OpenEdition}

Journals

Édition électronique

URL : http://journals.openedition.org/polysemes/1862

DOI : $10.4000 /$ polysemes. 1862

ISSN : 2496-4212

Éditeur

SAIT

Référence électronique

Jean-Michel Ganteau, «Logique de l'après-coup : Chatterton et le contemporain », Polysèmes [En ligne], 17 | 2017, mis en ligne le 30 avril 2017, consulté le 06 mai 2019. URL : http://journals.openedition.org/ polysemes/1862; DOI : 10.4000/polysemes. 1862

Ce document a été généré automatiquement le 6 mai 2019.

Polysèmes 


\title{
Logique de l'après-coup : Chatterton et le contemporain
}

\author{
The Logics of Afterwardsness: Presenting the Contemporary in Chatterton
}

Jean-Michel Ganteau

1 Chatterton est le quatrième roman de Peter Ackroyd, publié en 1987, au cœur des années Thatcher, et même s'il ne propose en rien une satire directe de la société britannique des années quatre-vingt, il n'en offre pas moins une pratique de l'intempestif, en ceci qu'il joue du décalage, ou plus précisément du «déphasage » temporel, pour citer Giorgio Agamben, se constituant ainsi en texte contemporain, et faisant d'Ackroyd un contemporain. Le récit retrace partiellement l'existence de Chatterton, célèbre faussaire, inventeur du passé et héraut du romantisme ; or, pour Agamben « celui qui appartient au contemporain, c'est celui qui ne coïncide pas parfaitement avec lui ni n'adhère à ses prétentions» (Agamben 2008, 9). Comme The Passion de Jeanette Winterson, publié la même année, Chatterton préfère les brumes du passé et les villes de l'intérieur à la City de Londres, les vivandières aux yuppies (Winterson, author's note, np.). Il s'agit d'un texte qui, empruntant les chemins de la biographie romancée et de la romance (Finney 258), revient vers le passé, à l'instar des métafictions historiographiques de l'époque, pour le subvertir ou à tout le moins le problématiser, et dans tous les cas le lier, pour faire de ce passé un parfait ou present perfect, afin d'en présenter et d'en exalter la présence, selon les termes de T.S. Eliot (Eliot 38). Ackroyd est en effet l'un des plus éliottiens des romanciers britanniques. Il a rédigé une biographie du maitre moderniste et a surtout repris les principes de T.S. Eliot dans un ouvrage de critique littéraire, publié dans les années soixante-dix, qui ressasse les principes de l'auteur de The Waste Land (Ackroyd 1976). À travers la lecture d'Agamben, j'entends donc ici le contemporain comme forme de l'inactuel nietzschéen, Nietzsche pour qui « exercer une influence inactuelle, [c'est] agir contre le temps, donc sur le temps, et, espérons-le, au bénéfice d'un temps à venir " (Nietzsche 94). C'est la figure historique de Chatterton, mais aussi le roman d'Ackroyd, dont il conviendra de souligner la contemporanéité. 
2 Pour ce qui est de l'après-coup, il s'agit d'un emprunt à Freud, « après-coup » étant la traduction plus ou moins heureuse de Nachträglichkeit, rendu en anglais par deferred action . La traduction anglaise, comme la version française, soulignent le décalage temporel, et le report, et ont souvent été associées au fonctionnement du trauma, tel qu'il est décrit en 1920 par Freud dans «Au-delà du principe de plaisir », par exemple. On se rappelle en effet que si l'on prend «trauma » dans son acception non commune, l'on fait référence à une effraction si violente qu'elle ne peut se connaître, que le patient achoppe à se la remémorer. Cette faillite de l'entendement, que Cathy Caruth a désignée en termes d'« insu » (ou « unknowing », Caruth 1996, 3) ou de "crise de la vérité » (ou « crisis of truth », Caruth 1995, 6), constitue l'une des caractéristiques essentielles du trauma. Et il convient de préciser qu'elle est toujours accompagnée d'un décalage qui affecte la mémoire et, partant, la linéarité de la mise en récit du souvenir. Dans le texte de 1920, Freud indique en effet que l'effraction violente déborde tellement le système de protection du patient que le souvenir en est bloqué, ce qui a des incidences en termes de linéarité : «[Le patient] est bien plutôt obligé de répéter le refoulé comme expérience vécue dans le présent au lieu de se le remémorer comme un fragment du passé, ce que préfèrerait le médecin " (Freud 57-58). En conséquence se manifeste une compulsion de répétition, qui toujours ramène le patient au point de fixation du traumatisme, en un "éternel retour du même " (Freud 62). Il est dès lors possible de voir la complexité de cette notion d'après-coup, qui suppose un élan vers l'ultérieur (les conséquences de l'effraction) alors même qu'elle contraint à un retour vers le moment de fixation, rendant impossible la mise en récit et affolant la chronologie, ainsi que le rappelle Jean Laplanche :

[L]e concept de Freud d'après-coup contient à la fois une grande richesse et une certaine ambiguïté, en ce qu'il combine une direction rétrogressive et une direction progressive. Je veux rendre raison de ce problème des directions différentes, vers l'avant et vers l'arrière, en argumentant que, dès le début, il y a quelque chose qui va dans la direction du passé vers le futur [...]. Ce message est alors retraduit, en suivant une direction temporelle tour à tour rétrogressive et progressive [...]. (Laplanche 1999, 66)

3 Or, il me semble que cette notion d'après-coup est à retenir dans notre analyse de l'intempestif, en ceci qu'elle permet d'éclairer un des fonctionnements du récit ackroydien, lorsqu'elle apparaît pour modifier les textes à venir mais aussi pour, selon le fameux paradoxe de T.S. Eliot dans «Tradition and the Individual Talent», modifier l'ensemble des œuvres du passé (Eliot 39-40). En ayant recours à cette catégorie, je suis conscient de faire converger deux notions appartenant à des domaines d'investigation hétérogènes, à savoir : la critique littéraire et la psychanalyse, et plus encore d'utiliser des catégories utilisées pour évoquer la singularité d'un patient afin de les appliquer à la singularité d'une œuvre ou à un domaine aussi vaste que celui de l'histoire littéraire. Cette migration n'a d'autre propos que d'éclairer un fonctionnement intempestif, à l'aide de mécanismes profonds de nature à proposer une lecture latérale du déphasage, et c'est pourquoi je me propose de m'intéresser à trois modalités de l'après-coup : comme retard, comme retour, comme puissance créatrice.

4 Avec Chatterton, comme dans nombre de ses récits, Ackroyd favorise un dispositif narratif fondé sur la multiplication des strates temporelles, en construisant un roman dans lequel le protagoniste contemporain est travaillé par le passé victorien (mettant en scène le peintre Wallis et le romancier Meredith, lequel sert de modèle pour le fameux tableau Chatterton). Il est également hanté par certains épisodes de la vie de Chatterton, qui 
fondent l'intrigue et la strate temporelle la plus ancienne, concernant la seconde partie du dix-huitième siècle. Pareille superposition, outre qu'elle favorise les correspondances et échos thématiques, multiplie les doublons, et fait vaciller les frontières ontologiques. Ainsi que l'ont repéré plusieurs commentateurs, le protagoniste de l'intrigue contemporaine, Charles Wychwood, est en effet tour à tour double de Meredith et de Chatterton. Par ailleurs, la mort du jeune poète, qui intervient en fin de récit, duplique celle, feinte et rétrospectivement reconstruite par Wallis, de la séance de pose, si bien que Chatterton mourant adopte le sourire hantant les lèvres de Meredith, dans le célèbre tableau visible à la Tate Gallery (Lanone 20). Par ailleurs, ainsi que le fait remarquer Catherine Lanone, la mort de Charles Wychwood est narrativement « cousue » à la scène de l'achèvement du tableau de Wallis, l'ensemble convergeant pour «suggérer que la signature noire de Wallis barre la vie de Charles, ou que c'est la mort de Charles qui confère au tableau sa présence, et sa tranquillité d'urne keatsienne » (Lanone 20). Ce qui caractérise la temporalité de Chatterton est, précisément, l'atemporalité (Finney 257), prenant la forme du cercle ou de la spirale (Onega 60) pour figurer un «mode anhistorique de l'écoulement du temps» (Bernard 5), ou figurer la co-présence et la simultanéité (Lanone 19), voire la co-temporalité (Onega 73). Cela revient en partie à désigner le présent comme archaïque, comme présence du passé, ce qui légitime les manifestations spectrales émaillant le roman, faisant se rencontrer en des anachronismes saisissants les personnages de strates temporelles diverses, sous forme de passages dont la dimension hallucinatoire est toujours perçue mais jamais totalement établie (Ackroyd 1987, 54, 70, 78 et passim), provoquant, dans les termes de Jean Laplanche, une " simultanéité de l'adulte et de l'enfant » (Laplanche 2006, 171).

5 C'est donc une logique du temps désajusté ou " maintenant désajointé », pour reprendre Derrida commentant Shakespeare (Derrida 1993, 21), que propose Chatterton. Le chronos se trouve ainsi subverti, et l'inexorablement chronologique devient temps de la liaison, temps de la hantise et du retour, en une présentation narrative de l'après-coup traumatique qui ordonne l'événement à un moment de fixation inaugural, et transforme la plupart des occurrences en récurrences. Comme dans la plupart des récits travaillés par la spectralité, c'est donc un sentiment de répétition et de retour permanent au moment de fixation traumatique (Freud 49, Ferenczi 20-21) qui affole le chronologique et désajointe le présent, la mort du protagoniste contemporain étant moins présentée comme occurrence unique que comme répétition ou reprise de celle de Chatterton, laquelle avait également été ré-éditée par le décès feint de Meredith campant celui que Wordsworth a baptisé "the marvellous Boy». Dans Chatterton, selon la logique traumatique de l'après-coup, le passé est donc moins remémoré que strictement répété, pour figurer un temps qui ne passe pas ou «temps mort» (Derrida 1967, 99). Outre que l'occurrence fait irruption, contrariant et trouant le temps chronologique, elle se constitue en événement, qui scelle la passivité du sujet diégétique, le désontologise, et le transforme par là même en figure du contemporain.

6 En effet, c'est précisément l'écart ou la non-coïncidence que subit le protagoniste de la strate temporelle la plus récente, Charles Wychwood, qui en fait l'image du contemporain, à savoir de celui qui adhère à son propre temps tout en prenant ses distances ou, en d'autres termes, de celui qui est sous l'effet de « la relation au temps qui adhère à lui par l'anachronisme et le déphasage » (Agamben 2008, 11). En d'autres termes, l'événement que constitue l'œuvre de Chatterton est présenté (et pas seulement représenté) comme moment de fixation, qui sans cesse affecte l'avenir et fait 
immanquablement signe à la postérité littéraire. Charles Wychwood, poète raté et visionnaire, artiste hanté par la tradition et radicalement affecté par le passé culturel et littéraire, devient de ce fait l'emblème du contemporain, lui qui, à l'instar de Chatterton, « fixe les yeux sur son propre temps pour en percevoir l'obscurité » (Agamben 2008, 19) et non point les aveuglantes lumières. Wychwood, tels Wallis et Chatterton, se laisse prendre par un échange de regards, et s'ordonne au présent " pour voir que l'obscurité de son temps le regarde et l'interpelle" (Agamben 22). Le temps devient donc site de l'anachronisme, de la coïncidence partielle et de l'inactuel qui travaille la chronologie de l'intérieur (Agamben 25-26) et au chronos préfère le kairos, signe de la liaison et de l'intempestif.

7 Or, le terme kairos est généralement utilisé pour évoquer un présent lié à un événement à venir, dans un contexte religieux, pour évoquer «le temps que le temps met pour finir " (Agamben 2000, 116-117), ce qui suggère une autre dimension de l'après-coup. En effet, le « ho nyn kairos » paulinien, s'il est le temps du maintenant, symptôme du contemporain, présente la caractéristique de transformer le temps, de le mettre en relation avec d'autres temps (Agamben 2008, 38). Cette relation entre les temps est différemment orientée, faisant du passé une prophétie du présent, et permet de se concentrer sur un sens particulier de l'après-coup, à savoir la modification de la première occurrence. C'est à tout le moins ce que rappelle Paola Mieli, commentant l'ambivalence de l'après-coup :

L'idée "d'action différée " implique la notion de déterminisme psychique, de décalage entre la stimulation et la réponse, ce qui suggère l'idée d'une décharge retardée. La notion freudienne de Nachträglichkeit va dans la direction opposée. Elle n'indique pas un décalage, un délai entre l'action et la réaction, mais au contraire un événement qui, dans l'acte de sa propre énonciation, réinvestit une inscription passée et acquiert le statut de révélation. Ce qui se passe «après » transforme ce qui l'a précédé. (Mieli, citée par Amfreville 75)

8 Au cœur de l'intrigue de Chatterton apparaît en effet une matrice policière dévoyée (Lanone 17), qui voit Charles Wychwood et son ami Philip retourner vers la figure historique de Chatterton et vers son œuvre afin de vérifier une hypothèse audacieuse : Chatterton ne se serait pas suicidé à dix-huit ans mais, mettant en scène sa mort, aurait continué à vivre et serait devenu le nègre d'auteurs comme Blake, voire les aurait inventés, de la même manière qu'il avait créé le moine Rowley en imaginant une œuvre $\mathrm{du} \mathrm{XV}^{\mathrm{e}}$ siècle, produisant l'un des canulars littéraires les plus retentissants de l'histoire culturelle britannique. Pareil mouvement de retour, mimant le sens de l'histoire littéraire, donne à entendre un autre sens de l'après-coup, lequel souligne que l'événement originel est lui-même soumis à modification, et que les manifestations ultérieures (actualisées par la lecture et par le commentaire) tendent à agir sur le passé. C'est vraisemblablement ce qui justifie les images d'ingestion et de consommation du passé, Wychwood léchant la poussière pour «manger le passé » (Ackroyd 1987, 15) et consommant les pages de Great Expectations (Ackroyd 1987, 49).

Or, le retour est inscrit au cœur du récit, et caractérise également la figure de Chatterton, lui dont l'œuvre a eu une influence sur la postérité romantique et moderne, alors que son inspiration l'a porté vers les brumes d'un passé dont il a sollicité la présence à travers la convocation intempestive du moine Rowley, dont le jeune poète aurait trouvé les textes. À ceci près que l'histoire littéraire nous apprend que Chatterton n'est pas l'inventeur au sens archéologique de Rowley, à savoir d'un individu, mais bien d'une époque, dans la mesure où son génie est censé avoir résidé dans le pastiche de la langue d'une époque, et dans la création de toutes pièces d'une figure fictive de l'auteur. Dès lors, ce que permet 
d'éclairer la catégorie de l'après-coup, dans sa dimension rétrospective, est la notion éliottienne de tradition, étroitement associée à ce que le poète moderniste qualifie de sens historique. On se rappelle en effet que ce sens historique se fonde sur la vision d'une présence simultanée de tous les auteurs, passés et présents, ce qui mène Eliot à proposer, en un paradoxe devenu célèbre, que l'introduction d'une nouvelle œuvre modifie l'ordre de la tradition. Il conclut en évoquant une inversion, en des termes qui sont passés à la postérité: "le passé devrait être modifié par le présent autant que le présent est déterminé par le passé $»^{1}$ (Eliot 39). À travers la figure historique et fictionnelle de Chatterton, c'est une littéralisation de cette proposition qui est fournie, dans la mesure où le jeune poète inventeur, en inventant le passé, bouleverse l'ordre de la tradition de manière radicale. En outre, ainsi que le rappelle Eliot, l'artiste du présent, de par son sens du temporel et de l'atemporel, devient conscient de sa place dans la tradition, et, partant, de sa contemporanéité :

Ce sens historique, qui se reconnait de l'atemporel comme du temporel, et de l'atemporel et du temporel tout à la fois, est précisément ce qui rend un auteur traditionnel. Et c'est en même temps ce qui rend un auteur conscient de sa situation dans le temps, de son époque et de sa propre contemporanéité. (Eliot 39)

10 Non seulement le contemporain regarde le passé et est regardé par le passé, mais encore il agit sur le passé, en une vision radicale de l'anachronique et de l'inactuel qui semble pousser un cran plus loin la vision du contemporain comme à la fois inscrit dans le présent et tout entier attaché à le désigner comme archaïque (Agamben 2008, 31).

11 Si l'on pousse ce raisonnement plus avant, il est possible de se rendre compte que ce que présente Chatterton échappe en fait au dessein de l'après-coup - ou le radicalise peutêtre. Même si la Nachträglichkeit est avant toute chose retour vers une première occurrence, laquelle se trouve modifiée par la seconde, et si l'on peut considérer pertinent de voir pareil fonctionnement appliqué à l'histoire littéraire (dans le cas de reprises, ou de ce que Bloom a nommé « the anxiety of influence » notamment), la figure de Chatterton implique un fonctionnement différent. En effet, dans le cas du Chatterton historique, tout se passe comme s'il y avait reprise, à savoir, comme si Chatterton revenait vers une première œuvre, celle d'un moine du $\mathrm{XV}^{\mathrm{e}}$ siècle pour, en la mettant au jour ou en la pastichant, non seulement lui donner sa place, mais encore en modifier le statut et l'ordre, conformément à la description éliottienne. Il s'agirait ainsi d'un cas de modification du passé ou d'action sur le passé, relativement paradoxal, mais aussi passablement pris en compte et expliqué par la critique d'inspiration éliottienne. Cela étant, comme précédemment indiqué, il apparaît que ce modèle est mis à mal par l'exemple de Chatterton. En effet, ce n'est point un retour vers une première occurrence identifiable qui est en jeu dans l'œuvre de l'enfant de Bristol, mais bien le retour vers un vide, vers une première occurrence fictive, qui n'a jamais existé en tant que telle, mais qui est le produit d'une imagination et d'une époque. En effet, en inventant de toutes pièces le moine Rowley, en le convoquant à grand renfort de pastiche, et en prenant comme modèle le style d'une époque et non d'un auteur, Chatterton répond à cet idéal d'impersonnel - qui sera théorisé par les modernistes, et notamment par Eliot - en devenant pur medium, et en privilégiant la reddition du soi pour laisser résonner la petite musique du passé, ce qu'Ackroyd a ailleurs qualifié de «musique d'Albion ». À travers ce canular littéraire, ce que fait Chatterton consiste moins à modifier le passé qu'à l'inventer, dans une redécouverte radicale du fonctionnement de l'après-coup, ce qu'Ackroyd souligne au cours d'un passage où Meredith et Wallis échangent leurs opinions sur les finalités de l'art : 
Mais Chatterton ne s'est pas borné à créer un individu. Il a inventé toute une période et en a adopté tout l'imaginaire: personne n'avait correctement compris le monde médiéval avant que Chatterton ne le fasse advenir à l'existence. Le poète ne se contente pas de recréer le monde ou de le décrire. Il le crée véritablement. Et c'est pour cela qu'il est craint. (Ackroyd 1987, 157) ${ }^{3}$

En ces lignes qui exaltent le pouvoir de la poiesis, il m'apparaît qu'est cernée au plus près la force de l'intempestif, lequel fait advenir ce qui n'était pas (si l'on me passe le pléonasme), créant du nouveau à partir de l'existant, en une version du creative borrowing ou emprunt créatif recommandé par Eliot. Dans ce cas d'un retour vers l'inexistant, l'après-coup se place au-delà de la modification, radicalise l'inversion temporelle pour ne plus faire apparaître que l'événement de la création qui, pris dans la logique de l'aprèscoup, est toujours déjà là, se borne à revenir dans son irréversibilité, sous l'effet de la répétition.

Ce que le roman d'Ackroyd s'attache à montrer ou à présenter, en fin de compte, c'est que l'après-coup est à l'œuvre en divers sens et directions, le geste de création devenant fausse récurrence, montrant de manière radicale que la création est toujours déjà reprise et annoncée, exacerbant la logique de l'après-coup, et proposant une vision de l'événement créatif comme toujours inactuel. Chatterton apparaît dès lors comme la monstrueuse présentation du contemporain, monstrueuse en ceci qu'elle donne à voir le contemporain et le met en ostension en jouant sur le décalage, sur le paradoxe de l'appartenance au présent et du rejet de ce présent. Force de répétition, force de retour et force de création tout à la fois, l'après-coup semble être au cœur de ce qui peut être considéré comme le plus éliottien des romans d'Ackroyd, tout entier consacré à l'évocation de la tradition et d'un aspect essentiel de l'anglicité, tout entier attaché à l'évocation du contemporain comme déphasage temporel.

\section{BIBLIOGRAPHIE}

Ackroyd, Peter. Notes for a New Culture (1976). London: Atkin Books, 1993.

Ackroyd, Peter. T.S. Eliot (1984). Harmondsworth: Penguin, 1985.

Ackroyd, Peter. Chatterton (1987). Harmondsworth: Penguin, 1993.

Agamben, Giorgio. Le Temps qui reste. Un commentaire de l'Épître aux Romains. Trad. Judith Revel.

Paris : Payot, 2000.

Agamben, Giorgio. Qu'est-ce que le contemporain? Paris : Payot, 2008.

Amfreville, Marc. Écrits en souffrance. Paris : Houdiard, 2009.

Bernard, Catherine. « Peter Ackroyd entre plagiat et élégie ». Études britanniques contemporaines 5

(mai 1994) : 13-22.

Caruth, Cathy (ed). Trauma: Explorations in Memory. Baltimore and London: Johns Hopkins UP, 1995. 
Caruth, Cathy. Unclaimed Experience. Trauma, Narrative, and History. Baltimore and London: Johns Hopkins UP, 1996.

Derrida, Jacques. De la grammatologie. Paris : Éditions de Minuit, 1967.

Derrida, Jacques. Spectres de Marx. Paris : Galilée, 1993.

Eliot, T.S. « Tradition and the Individual Talent ». The Sacred Wood. Essays on Poetry and Criticism (1920). London: Faber, 1997, 38-42.

Ferenczi, Sandor. Le Traumatisme (1982). Paris : Payot, 2006.

Freud, Sigmund. « Au-delà du principe de plaisir » (1920). Essais de psychanalyse. Paris : Payot, 1981, 43-114.

Ganteau, Jean-Michel. Peter Ackroyd et la musique du passé. Paris : Houdiard, 2008.

Lanone, Catherine. «Cryptes intertextuelles : jeux de lecture dans Chatterton de Peter Ackroyd ». Études britanniques contemporaines 12 (décembre 1997) : 17-30.

Laplanche, Jean. Entre séduction et inspiration : l'homme. Paris : PUF, 1999.

Laplanche, Jean. Problématiques IV. L'Après-coup. Paris : PUF, 2006.

Mieli, Paola. « Le temps du traumatisme ». Actualités de l'hystérie. Toulouse : Eres, 2001.

Nietzsche, Friedrich. Considérations inactuelles I et II. Eds. G. Colli et M. Montinari. Traduit de l'allemand par Pierre Rusch. Paris : Gallimard, 1990.

Onega, Susana. Metafiction and Myth in the Novels of Peter Ackroyd. Columbia, SC: Camden House, 1999.

Roudinesco, Élisabeth et Michel Plon. Dictionnaire de la psychanalyse. Paris : Fayard, 2006.

Winterson, Jeanette. The Passion (1987). London: Vintage, 1996.

\section{NOTES}

1. «[T]hat the past should be altered by the present as much as the present is directed by the past. »

2. "This historical sense, which is a sense of the timeless as well as of the temporal and of the timeless and of the temporal together is what makes a writer traditional. And it is at the same time what makes a writer most acutely conscious of his place in time, of his own contemporaneity. "

3. «But Chatterton did not create an individual simply. He invented an entire period and made its imagination his own: no one had properly understood the medieval world until chatterton summoned it into existence. The poet does not merely recreate or describe the world. He actually creates it. And that is why he is feared. » 


\section{RÉSUMÉS}

Cet article propose une lecture du roman de Peter Ackroyd à la lumière d'un des concepts clés de la psychanalyse et plus particulièrement de la théorie du trauma: l'après-coup. Plus spécifiquement, il s'inspire du mouvement éliottien d'inversion temporelle et de modification rétrospective du canon qu'Ackroyd met en scène dans son récit à travers la figure de Chatterton écrivant (ou inventant) le passé littéraire pour s'intéresser à la double temporalité critique de l'après-coup. Cette dernière permet de situer le contemporain comme moment intempestif, et c'est ce que l'article s'attache à faire en examinant les catégories du retard, du retour, et de la création.

In this article I provide a reading of Peter Ackroyd's novel in the light of one of the key concepts of psychoanalysis in general, and more particularly of trauma theory, i.e. afterwardsness. I build up on the Eliottian vision of the inversion of temporality, when the introduction of a work of art modifies the whole of the tradition. Ackroyd stages such an inversion in his fictional biography of Chatterton who wrote (invented) the literary past into existence, which I choose to read in the light of the double, paradoxical temporality of aferwardsness. Such a reading allows for a vision of the contemporary as the site of the disjointed and untimely through the categories of belatedness, return and creation.

\section{INDEX}

oeuvrecitee Chatterton

Keywords : afterwardsness, belatedness, contemporary, creation, kairos, return, time, untimely Mots-clés : après-coup, contemporain, création, intempestif, kairos, retard, retour, temps

\section{AUTEURS}

\section{JEAN-MICHEL GANTEAU}

Jean-Michel Ganteau est professeur de littérature anglaise à l'Université Paul-Valéry Montpellier. Il dirige la revue Études britanniques contemporaines. Il est l'auteur de deux monographies, David Lodge: le choix de l'éloquence (PU de Bordeaux, 2001) et Peter Ackroyd et la musique du passé (Houdiard, 2008). Il a également publié, en collaboration avec Christine Reynier, quatre volumes aux Presses Universitaires de la Méditerranée : Impersonality and Emotion in Twentieth-Century British Literature (2005), Impersonality and Emotion in Twentieth-Century British Arts (2007), Autonomy and Commitment in Contemporary British Literature (2010) et Autonomy and Commitment in Contemporary British Arts (2012) ; en collaboration avec Susana Onega, il a publié The Ethical Component in Experimental British Fiction since the 1960s (Cambridge Scholars Publishing, 2007), Trauma and Ethics in Contemporary British Literature (Rodopi, 2011), Trauma and Romance in Contemporary British Literature (Routledge, 2012), Contemporary Trauma Narratives: Liminality and the Ethics of Form (Routledge, 2014) et Victimhood and Vulnerability in Twenty-first Century Fiction 
(Routledge, 2017). Il a également publié de nombreux articles sur le roman britannique contemporain, s'intéressant particulièrement à l'éthique des affects. Son ouvrage intitulé The Ethics and Aesthetics of Vulnerability in Contemporary British Fiction a paru dans la collection Contemporary Literature chez Routledge en 2015. 